\title{
Analysis of the Effect of Various Methods Providing Uniform Load Distribution to a Mangle
}

\author{
Part 1: Surface Cambering, Moment Compensation, and Intermediate Support \\ By Kumeo Nakajima and Shozo Tsujio, Members, TMSJ \\ Department of Mechanical Engineering, University of Osaka Prefecture, Osaka
}

Based on the Journal of the Textile Machinery Society of Japan, Transactions, Vol. 19, No. 11, T274-283 (1966); Vol. 21, No. 6, T125-136 (1968)

\begin{abstract}
There are many ingenious methods to provide nip uniformity by making the load distribution of a mangle uniform. In this paper the authors develop mathematical analysis of the effect of three such methods. The first is surface cambering which compensates the roller deflection by the distributed thickness of the roller covering along the length of the covered rollers. The second is moment compensation which applies a moment to each end of the roller shaft tending to deflect the covered roller in the opposite direction to that due to normal deflection. The last is a method in which the covered rollers are supported at some intermediate positions between the ends and the center, instead of at both ends.

The fundamental theory of the bending of symmetrical rubber-covered pressure rollers derived in the earlier paper is applied to these three cases mentioned above. The following is a summary of the conclusions obtained from theoretical analysis and numerical calculations:

(1) Surface cambering providing a rigorously uniform load distribution against a certain design load is the same as the deflection of the roller mandrel due to the uniformly distributed load which is equal to that design load.

(2) Once surface cambering is determined for one loading condition of a mangle, the load distribution becomes uneven when the mangle works under other loading conditions.

(3) Theoretical analysis of moment compensation method has revealed that there exists an optimum amount of the compensating moment which is proportional to a moment $P L$ and that the moment is almost independent of $\lambda$ below 1.0. The covered rollers on which exerts the optimum compensation moment excel the clamped-support covered rollers in nip uniformity.

(4) Intermediate-support covered rollers are the best in nip uniformity when they are supported at an optimum position. This optimum position is about $55 \%$ of half of the length of the covered rollers from the center, and is independent of the characteristic number $\lambda$ and of the average load $\bar{w}$.
\end{abstract}

KEY WORDS: BENDING, BENDING MOMENTS, CAMBER, COMPENSATION, COVERED ROLLS, DEFLECTION, MANGLES, PRESSURE DISTRIBUtion, PRESSURe ROLLS, ROLL COVERINGS, ROLL CROWNS, SQUEEZE ROLLS, UNIFORMITY

\section{Introduction}

Nip uniformity is the most important requirement for the modern squeezing and padding mangles. To provide more uniform load distribution along the working length of the covered rollers, many ingeneous mechanisms are developed and used in practice.

In this paper the authors analyze the effect of the following three types of uniform pressure mechanisms theoreti- cally, making use of the fundamental theory on symmetric bending of a pair of mechanically similar rubber-covered pressure rollers described in the earlier paper ${ }^{[1]}$ :

(1) Using rubber covering with surface camber in which the thickness of the covering varies along the length of the covered roller.

(2) Applying a moment to each end of the roller shaft tending to deflect the covered roller in the opposite direc- 
tion to that due to normal deflection.

(3) Supporting the covered rollers at some intermediate positions between the center and the ends of the covered rollers, instead of at their both ends.

\section{Nomenclatures}

In this paper the following nomenclatures are used:

$A=$ Integration constant

$A_{C}=$ Constant concerning clamped-support covered rollers

$A_{H}=$ Constant concerning hinged-support covered rollers

$B=$ Integration constant

$\boldsymbol{B}_{C}=$ Constant concerning clamped-support covered rollers

$B_{H}=$ Constant concerning hinged-support covered rollers

$C, D=$ Integration constants

$E, E_{1}, E_{2}=$ Young's moduli of material of which roller mandrel is made

$F, F^{\prime}=$ Integration constants

$f=$ Symbol of function

$G, G^{\prime}, H, H^{\prime}=$ Integration constants

$I, I_{1}, I_{2}=$ Secondary moments of sectional area of roller mandrel around neutral axis

$J, J^{\prime}=$ Integration constants

$K=$ Stiffness of mangle frame

$k=$ Constant which expresses elastic property of roller covering

$2 L=$ Working width of covered roller

$2 L_{2}=$ Distance between supports $\left(L_{2}\right.$ shorter than $\left.L\right)$

$M=$ Bending moment

$M_{C}=$ Clamping moment

$2 P=$ Total force acting on covered roller

$u=$ Thickness of covering

$u_{c}=$ Uniform thickness of covering

$u_{L}=$ Thickness of covering at $x=L$

$u_{0}=$ Thickness of covering at $x=0$

$w=$ Distributed load per unit length of covered roller

$\bar{w}=P / L$

$w_{\text {des }}=$ Value of $\bar{w}$ for design load

$w_{C}=$ Distributed load per unit length of clamped-support covered roller

$w_{H}=$ Distributed load per unit length of hinged-support covered roller

$x=$ Distance along center line of undeformed covered roller

$y=$ Deflection of roller mandrel

$\bar{y}=\bar{w} /\left(k / u_{c}\right)$

$y_{C}=$ Deflection of clamped-support roller mandrel

$y_{H}=$ Deflection of hinged-support roller mandrel

$y_{0}=$ Deflection of roller mandrel at $x=0$

$z=$ Thickness of deformed covering

$z_{L}=$ Thickness of deformed covering at $x=L$

$\alpha=K L / E I$

$\beta=E_{2} I_{2} / E_{1} I_{1}$ $\gamma=1-w_{\text {des }} / k$

$\varepsilon=w-\bar{w}$

$\zeta=z / \bar{y}$

$\eta=u_{L} / y_{0}$

$\kappa=\bar{w} / w_{\text {des }}$

$\lambda=\left\{\left(k / u_{c}\right) /\left(4 E I / L^{4}\right)\right\}^{1 / 4}$

$\lambda_{L}=\left\{\left(k / u_{L}\right) /\left(4 E I / L^{4}\right)\right\}^{1 / 4}$

$\lambda_{0}=\left\{\left(k / u_{0}\right) /\left(4 E I / L^{4}\right)\right\}^{1 / 4}$

$\lambda_{1}=\left\{\left(k / u_{c}\right) /\left(4 E_{1} I_{1} / L^{4}\right)\right\}^{1 / 4}$

$\lambda_{2}=\left\{\left(k / u_{c}\right) /\left(4 E_{2} I_{2} / L^{4}\right)\right\}^{1 / 4}$

$\mu=M / M_{C}$

$\mu_{o p t}=$ Optimum value of $\mu$ which provides the most uniform load distribution

$\xi=x / L$

$\sigma=L_{2} / L$

$\phi, \phi=$ Symbols of function

\section{Thickness Distribution of Covering Which Provides a Uniform Load Distribution}

Very few investigations are known which dealt with the optimum thickness distribution of covering. One of them solved the problem by trial and error method ${ }^{[2]}$, and another one ignored the presence of the covering ${ }^{[3]}$.

The basic equations derived by the authors make it possible to determine the optimum thickness distribution analytically without relying on any approximations except the assumptions adopted. Under the same assumptions as in Section 3 of the earlier paper ${ }^{[1]}$, the basic equations of the bending of the rubber-covered pressure rollers are:

$$
\begin{aligned}
& E I\left(d^{4} y / d x^{4}\right)=w \\
& w=(k / u)(u-z) \\
& y=z-z_{L}
\end{aligned}
$$

As the load distribution is expected to be uniform, eq. (3.1) is reduced to

$$
E I\left(d^{4} y / d x^{4}\right)=w_{d e s}=P / L
$$

Eq. (3.4) is solved, modifying the general boundary conditions expressed by eq. (4.12) of the earlier paper ${ }^{[1]}$, namely:

$$
\left.\begin{array}{cl}
y^{\prime}=0 & \text { at } x=0 \\
y=0, E I y^{\prime \prime}=-K y^{\prime}, E I y^{\prime \prime \prime}=P & \text { at } x=L
\end{array}\right\} \ldots
$$

The solution in dimensionless expression is given by

$$
y / y_{0}=\left\{\left(1-\xi^{2}\right)\left(5-\xi^{2}\right)+c\left(1-\xi^{2}\right)^{2}\right\} /(1+\alpha) .
$$

where

$$
\begin{aligned}
& y_{0}=y(0)=w_{d e s} L^{4} / 24 E I \\
& \xi=x / L
\end{aligned}
$$

$\alpha$ is a stiffness ratio, which is a measure of the stiffness of the mangle frame and the bearing box. When $\alpha=0$, the support is a hinged one, and when $\alpha=\infty$, it is a clamped one. Eq. (3.6) indicates that when the covered rollers are supported elastically, the deflection is expressed as a 
weighted mean of the above two extreme cases, $\alpha$ being the weight.

From eqs. (3.2), (3.3) and (3.6), the camber distribution is calculated:

$$
\begin{gathered}
\left(u-u_{L}\right) / y_{0}=(1 / \gamma)\left(y / y_{0}\right)=(1 / \gamma)\left\{\left(1-\xi^{2}\right)\left(5-\xi^{2}\right)\right. \\
\left.\quad+\alpha\left(1-\xi^{2}\right)^{2}\right\} /(1+\alpha) \quad \ldots \ldots \ldots \ldots \ldots \ldots \ldots \ldots \ldots \ldots \ldots \ldots \ldots \ldots \ldots \ldots \ldots \ldots \ldots
\end{gathered}
$$

where

$$
\gamma=1-\left(w_{\text {des }} / k\right),
$$

$w_{d e s} / k$ being the compressive strain induced in the covering of unit thickness and of unit length of the covered rollers. In other words, $\gamma$ is a ratio of the thickness of the covering after and before the deformation.

Putting $\xi=0$ in eq. (3.10),

$$
\left(u-u_{L}\right)_{\max } / 2 L=\left(y_{0} / 2 L\right)(1 / \gamma)(5+\alpha) /(1+\alpha)
$$

The above discussions are for the covered rollers supported at both ends. If they are supported at the center, the boundary conditions (3.5) should be replaced by the following equations, modifying eq. (4.21) of the earlier paper $^{[1]}$,

$$
\begin{aligned}
& y^{\prime}=0, \quad y^{\prime \prime \prime}=-P / E I \\
& y=0, \quad y^{\prime \prime}=0 \\
& \text { at } x=0_{+} \\
& \text {at } x=L\}
\end{aligned}
$$

where 0 means that $x \rightarrow 0$ keeping $x \geqq 0$.

The solution of eq. (3.4) in dimensionless form is:

$$
y / y_{0}=-3+6 \xi^{2}-4 \xi^{3}+\xi^{4}, \quad \xi \geqq 0
$$

The camber distribution and the maximum camber are given by

$$
\begin{aligned}
& \left(u-u_{L}\right) / y_{0}=(1 / \gamma)\left(-3+6 \xi^{2}-4 \xi^{3}+\xi^{4}\right), \quad \xi \geqq 0 \ldots \\
& \left(u_{L}-u\right)_{\max } / 2 L=\left(y_{0} / 2 L\right)(3 / \gamma) \ldots \ldots \ldots \ldots \ldots \ldots \ldots \ldots \ldots \ldots \ldots \ldots \ldots
\end{aligned}
$$

The right hand side of eq. (3.6) expresses the deflection of a uniformly loaded beam with elastic support, because it is derived without using eqs. (3.2) and (3.3). Since eq. (3.2) is modified into $u=z / \gamma$, the thickness of undeformed covering is given by multiplying the thickness of deformed covering with $1 / \gamma$. Thus the thickness of the compressed covering is converted into its natural thickness. Camber is a difference between the thickness of the covering at any point and that at the end. Hence the camber distribution is expressed as a product of deflection and $1 / \gamma$.

The expression of the optimum camber distribution contains $u_{L}$ undetermined. From this fact it is concluded that the distribution of the absolute value of the thickness does not determine the uniform load distribution, but that the camber, or roller crown, determine the uniform load distribution. Hence the absolute value of the thickness of the covering has a freedom to be arbitrarily chosen by considering the maximum pressure or the nip width.

The results of the numerical calculation of the optimum camber distribution are shown in Fig. 1. If the constant $\alpha$ is smaller than 1 , the camber distribution of the covering of the elastically supported rollers is almost the same as that of the hinged-support rollers. It is clear from eq. (3.12) that

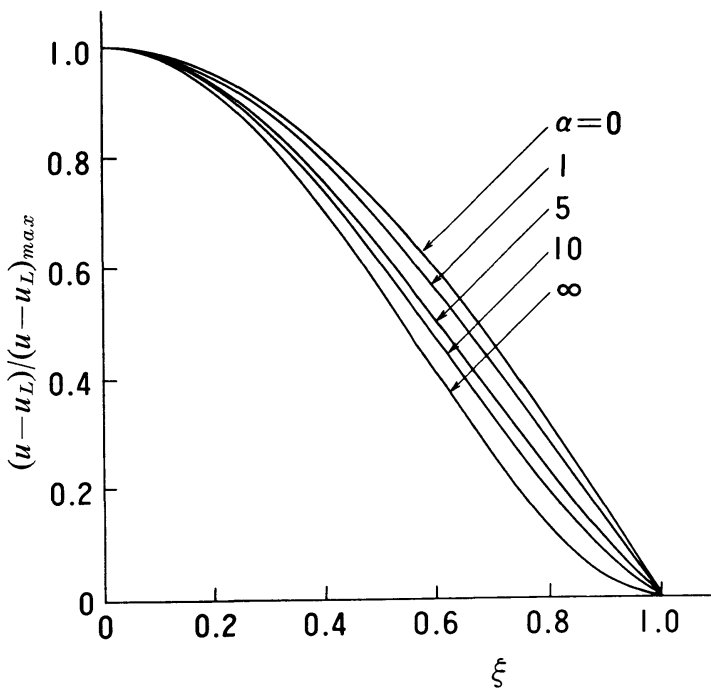

Fig. 1 Camber Distribution Providing Uniform Load Distribution

the dimensionless maximum camber $\left(u-u_{L}\right)_{\max } / 2 L$ is determined not only by the design load and the elastic property of the covering, but also by the support condition of the rollers, when the roller mandrel is given. Again, the dimensionless maximum camber is approximately equal to that of the hinged-support rollers when $\alpha<1$.

\section{The Load Distribution when Rollers with Covering of the Optimum Camber Distribution are used under the Loads Other than the Design Load}

The investigation of the previous section indicates that the uniform load distribution is realized only when the covered rollers with the optimum camber distribution are used under the design load.

In practice a mangle does not always process the same kind of fabric under the same load. Therefore, it is not of no value to investigate how the uniformity of load distribution is lowered when the actual force acting on the covered rollers differs from the force corresponding to the design load.

When the thickness of the covering is a function of $x$, an equation:

$$
E I\left(d^{4} z / d x^{4}\right)+(k / u) z=k
$$

is derived from the basic equations (3.1), (3.2) and (3.3). This is no longer a linear differential equation with constant coefficients, but that with variable constants.

This equation can be solved by several methods such as series expansion method, energy method and numerical method. Here the last two methods were tried first, and almost the same results were obtained. Thus the rest of the calculation is carried out numerically making use of an electronic digital computer. 

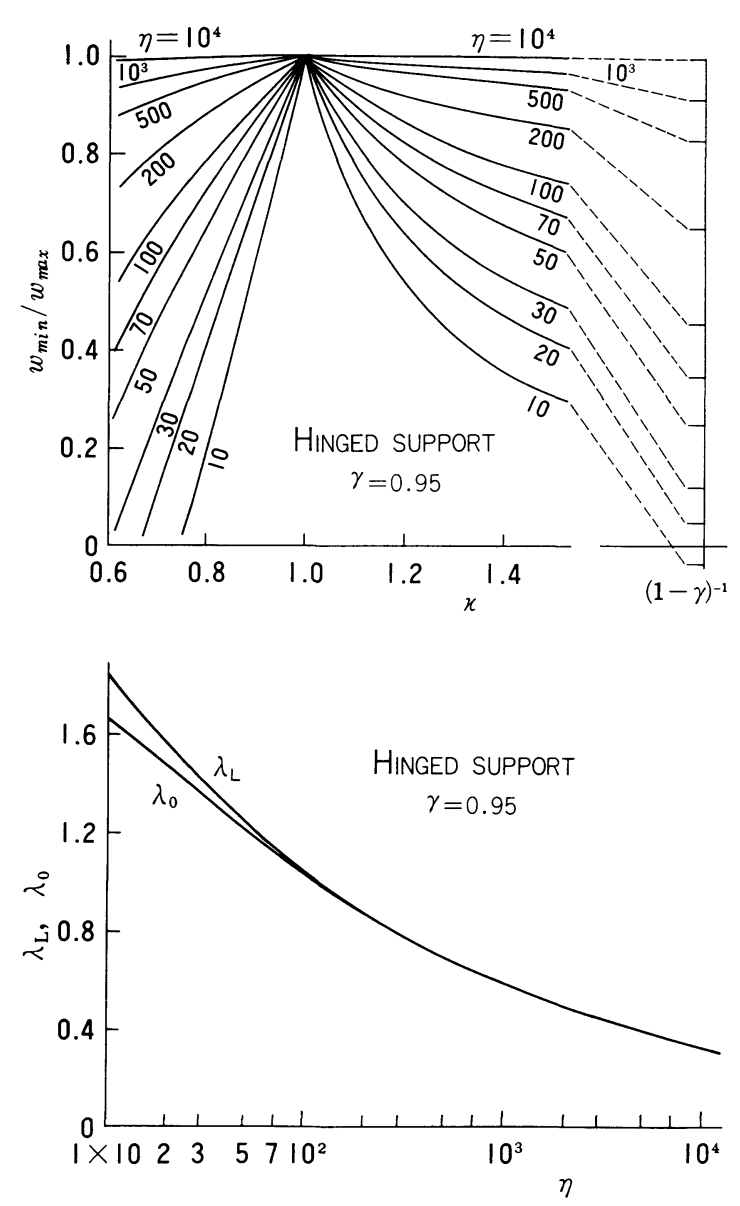

(a)
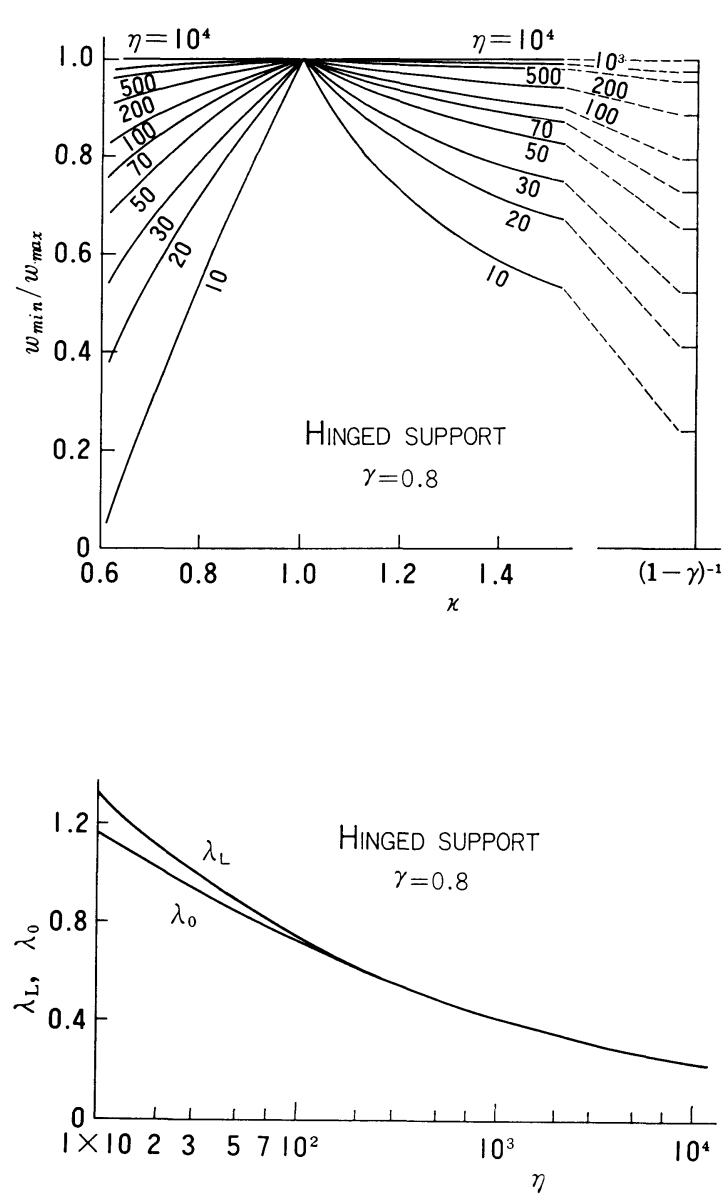

(b)

Fig. 2 Effect of Mean Load on Uniformity Index of Load Distribution of Cambered Rollers, Loading Condition Being Different from Design Condition

If eq. (3.10) of the optimum thickness distribution is substituted into eq. (4.1),

$$
d^{4} \zeta / d \xi^{4}+4 \lambda_{L}{ }^{4}\{1+f(\alpha, \gamma, \xi) / \eta\}^{-1 \zeta}=4 \lambda_{L} L^{4} /\{r(1-\gamma)\}
$$

is obtained. Here

$$
\begin{aligned}
& f(\alpha, \gamma, \xi)=(1 / \gamma)\left\{\left(1-\xi^{2}\right)\left(5-\xi^{2}\right)+\alpha\left(1-\xi^{2}\right)^{2}\right\} /(1+\alpha) \\
& \lambda_{L}{ }^{4}=\left(k / u_{L}\right) /\left(4 E I / L^{4}\right)=6 /\{\eta(1-\gamma)\} \\
& \kappa=\bar{w} / w_{\text {des }} \\
& \eta=u_{L} / y_{0}
\end{aligned}
$$

It should be noticed that $\zeta$ is defined by putting $u_{c}=u_{L}$ in eq. (4.2).

Two boundary conditions are almost the same as eq. (4.13) of the previous paper ${ }^{[1]}$. Namely:

$$
\ddot{\zeta}=-\alpha \dot{\zeta}, \quad \dddot{\zeta}=4 \lambda_{L}{ }^{4} \quad \text { at } \quad \xi=1
$$

From the symmetric property of the deformation the other two conditions are written as follows:

$$
\dot{\zeta}=0, \quad \dddot{\zeta}=0 \quad \text { at } \quad \xi=0 . .
$$

Three independent parameters $\eta, \gamma, \kappa$ are contained in the fundamental equation and three independent parmeters $\alpha, \eta, \gamma$ in the boundary conditions.

One of the typical results of the numerical calculation of the uniformity index of load distribution is shown in Fig. 2. Fig. 2(a) is a diagram for hinged-support rollers, the covering of which is compressed by $5 \%$, and Fig. 2(b) is the one for $20 \%$ compression. In each diagram is added a conversion chart from $\eta$ to $\lambda_{L}, \lambda_{0}$, respectively.

As these diagrams explain, the deterioration of the uniformity index of load distribution is less marked when thicker covering is compressed by a greater amount and when the actual mean load is higher than the design load.

More important is the fact that the uniformity of load distribution is injured considerably when the mean load acting on the covered rollers differs from the design load, particularly when the former is lower than the latter. Hence the cambered covered rollers should be used under the operating conditions rigorously controlled. 
Another interesting fact is recognized from Fig. 2. When the actual mean load increases, the covering is compressed to become thinner. The value of $\kappa$ when the thickness of the covering after deformation is zero is calculated. It is found that the value of $\kappa$ is equal to the reciprocal of $(1-\gamma)$, namely, to the reciprocal of the strain of the covering for the design load. The uniformity index of load distribution for $\kappa=(1-\gamma)^{-1}$ is always smaller than that for $\kappa>1$.

Fig. 2 also explains the relationship between $\lambda_{L}$ and $\eta$ of the covered rollers of uniform thickness of $u_{L}$, and the relationship between $\lambda_{0}$ and $\eta$ of the covered rollers of uniform thickness of $u_{0}$. The calculation of the uniformity index of load distribution of these covered rollers of uniform thickness reveals that the uniformity index of load distrinution of the covered rollers with the optimum camber for the value of $\kappa=(1-\gamma)^{-1}$ is greater than that of the covered rollers with uniform thickness of $u_{L}$, and that it is smaller than that of the covered rollers with uniform thickness of $u_{0}$. The difference between the uniformity index of load distribution of the covered rollers with uniform thickness of $u_{L}$ and that of the covered rollers with uniform thickness of $u_{0}$ is small and it is almost zero in the range of $\eta>100$ as Fig. 2 indicates. Hence the optimum cambering should be determined by the smallest mean load when the mangle does not work under a single loading condition, but works under several loading conditions.

\section{Theoretical Analysis of Moment Compensation Method}

An ingenious mechanism providing uniform load distribution is invented to apply some moment at each end of the covered rollers, tending to deflect the covered rollers in the opposite direction to that due to normal bowing deflection. From the view point of mechanics this type of covered rollers is assumed to be hinged-support beam at both ends of which act shearing forces and bending moments as shown in Fig. 3. For the simplicity of calculation and from the practical consideration the thickness of the covering is assumed to be uniform.

Borrowing the results of the earlier paper ${ }^{[1]}$, the basic equations are expressed by eqs. (3.1), (3.2) and (3.3) and

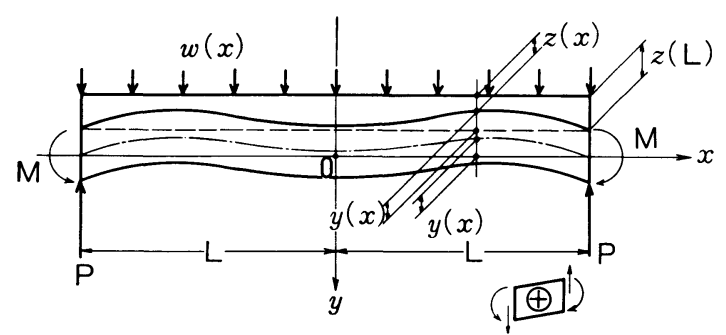

Fig. 3 Equivalent Mechanical Model of Covered Rollers, Compensating Moment Being Applied at Each End $u=u_{c}$. The boundary conditions should be modified:

$$
y^{\prime \prime}=M / E I, \quad y^{\prime \prime \prime}=P / E I \quad \text { at } \quad x=L
$$

In dimensionless form, eq. (5.1) is replaced by

$$
\dddot{\zeta}=4 \lambda^{4}(M / P L), \quad \dddot{\zeta}=4 \lambda^{4} \quad \text { at } \quad \xi=1 \text {. }
$$

The fundamental equation which determines $\zeta$ is written as

$$
d^{4} \zeta / d \xi^{4}+4 \lambda^{4} \zeta=4 \lambda^{4}\left(u_{c} / \bar{y}\right) \text {. }
$$

The solution of eq. (5.3) which satisfies the boundary conditions (5.2) is given by the following equations.

$$
\begin{aligned}
& \zeta=u_{c} / \bar{y}+A \dot{\psi}_{1}(\lambda \xi)+B \dot{\psi}_{2}(\lambda \xi) \\
& A=-\dot{\phi}_{1}(\lambda) / \phi_{61}(\lambda)-\mu\left\{\phi_{82}(\lambda) / \phi_{11}(\lambda)\right\}\left\{\phi_{2}(\lambda) / \phi_{61}(\lambda)\right\} \\
& B=-\dot{\phi}_{2}(\lambda) / \phi_{61}(\lambda)+\mu\left\{\phi_{82}(\lambda) / \phi_{11}(\lambda)\right\}\left\{\phi_{1}(\lambda) / \phi_{61}(\lambda)\right\} \\
& \mu=M / M_{C} \\
& M_{C}=(P L / 2) \cdot \phi_{82}(\lambda) /\left\{\lambda^{2} \phi_{11}(\lambda)\right\}
\end{aligned}
$$

Here, $\mu$ is a compensating moment coefficient, and $M_{C}$ is a clamp moment of the covered rollers with clamped support.

Substituting $z=\bar{y} \cdot \zeta$ into eqs. (3.2) and (3.3), the deflection and the load distribution of the covered rollers are obtained:

$$
\begin{aligned}
& y / \bar{y}=A\left\{\dot{\psi}_{1}(\lambda \xi)-\dot{\psi}_{1}(\lambda)\right\}+B\left\{\dot{\psi}_{2}(\lambda \xi)-\dot{\psi}_{2}(\lambda)\right\} \\
& w / \bar{w}=-\left\{A \dot{\psi}_{1}(\lambda \xi)+B \dot{\psi}_{2}(\lambda \xi)\right\} \quad \ldots \ldots \ldots \ldots \ldots \ldots \ldots \ldots \ldots \ldots \ldots
\end{aligned}
$$

It is evident that $\mu=1$ corresponds to the clamped support, and $\mu=0$ to the hinged support. Substituting $\mu=$ 1 and 0 into eq. (5.5), and designating the left hand sides of each equation by $A_{C}, B_{C}$ and $A_{H}, B_{H}$, respectively, the following equations are obtained.

$$
\left.\begin{array}{l}
A=\mu A_{C}+(1-\mu) A_{H} \\
B=\mu B_{C}+(1-\mu) B_{H}
\end{array}\right\}
$$

where

$$
\left.\begin{array}{ll}
A_{C}=-\psi_{1}(\lambda) / \phi_{11}(\lambda), & A_{H}=-\dot{\phi}_{1}(\lambda) / \phi_{61}(\lambda) \\
B_{C}=-\psi_{2}(\lambda) / \phi_{11}(\lambda), & B_{H}=-\dot{\phi}_{2}(\lambda) / \phi_{61}(\lambda)
\end{array}\right\}
$$

Substituting the above equations into eqs. (5.8) and (5.9),

$$
\begin{aligned}
& y / \bar{y}=\mu\left(y_{C} / \bar{y}\right)+(1-\mu)\left(y_{H} / \bar{y}\right) . \\
& w / \bar{w}=\mu\left(w_{C} / \bar{w}\right)+(1-\mu)\left(w_{H} / \bar{w}\right) .
\end{aligned}
$$

In these equations, suffices $C$ and $H$ signify the clamped support and the hinged support, respectively. $w_{C}, w_{H}, y_{C}$ and $y_{H}$ are derived from eqs. (4.17) and (4.18) of the earlier paper ${ }^{[1]}$.

Now, the compensating moment coefficient $\mu$ should be determined. The uniformity index is an excellent criterion of the nip uniformity for practical application. However, from the theoretical view point, the mean square of the deviation of the load distribution is more preferable, which is given by

$$
\overline{\varepsilon^{2}}=1 / 2 \int_{-1}^{1}(w-\bar{w})^{2} d \xi
$$


Substituting eq. (5.13) into eq. (5.14),

$$
\overline{\varepsilon^{2}}=\int_{0}^{1}\left\{w_{H}+\mu\left(w_{C}-w_{H}\right)\right\}^{2} d \xi-\bar{w}^{2}
$$

The optimum compensating moment coefficient $\mu$ is assumed to take a value which makes $\overline{\varepsilon^{2}}$ minimum. By partially differentiating eq. (5.15) by $\mu$ and putting the result zero:

$$
\frac{d}{d \mu} \overline{\varepsilon^{2}}=2 \int_{0}^{1}\left\{w_{H}+\mu\left(w_{C}-w_{H}\right)\right\}\left(w_{C}-w_{H}\right) d \xi=0 \ldots
$$

The optimum compensating moment coefficient is thus given by

$$
\mu_{O p t}=\frac{-\int_{0}^{1} w_{C} w_{H} d \xi+\int_{0}^{1} w_{H}{ }^{2} d \xi}{\int_{0}^{1} w_{C}{ }^{2} d \xi-2 \int_{0}^{1} w_{C} w_{H} d \xi+\int_{0}^{1} w_{H}{ }^{2} d \xi}
$$

\section{Numerical Results and Discussions}

The clamp moment, the optimum compensating moment coefficient and the dimensionless optimum compensating moment are plotted in Fig. 4 as functions of $\lambda$. The optimum compensating moment coefficient starts from a value of $9 / 7$ for $\lambda=0$, and increases rapidly for the value of $\lambda$ greater than 1. However, the optimum compensating moment decreases very slowly with an increase in $\lambda$. Thus it is almost independent of $\lambda$ for values of $\lambda$ below 1 .

Load distribution diagrams when the optimum compensating moments are applied are shown in Fig. 5 for five values of $\lambda$. The uniformity index of load distribution vs. the characteristic number is plotted in Fig. 6 from the data

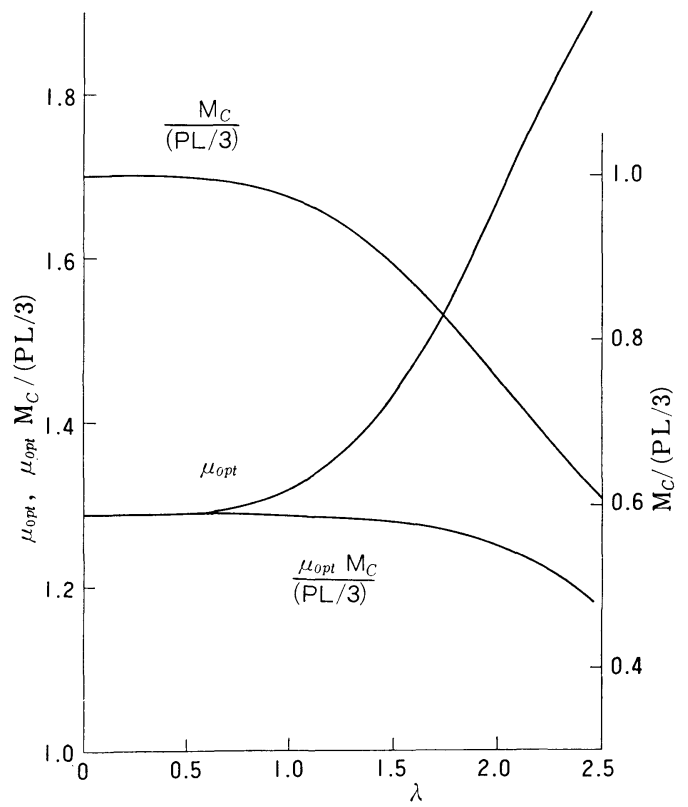

Fig. 4 Clamp Moment, Optimum Compensating Moment Coefficient and Dimensionless Optimum Compensating Moment

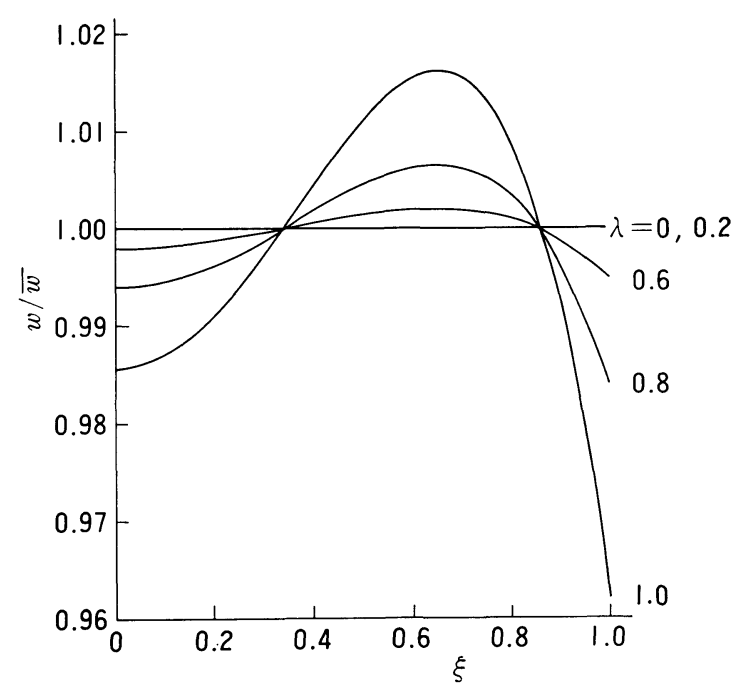

Fig. 5 Load D..$\underline{2}$ ribution, the Optimum Compensating Moment Being Applied

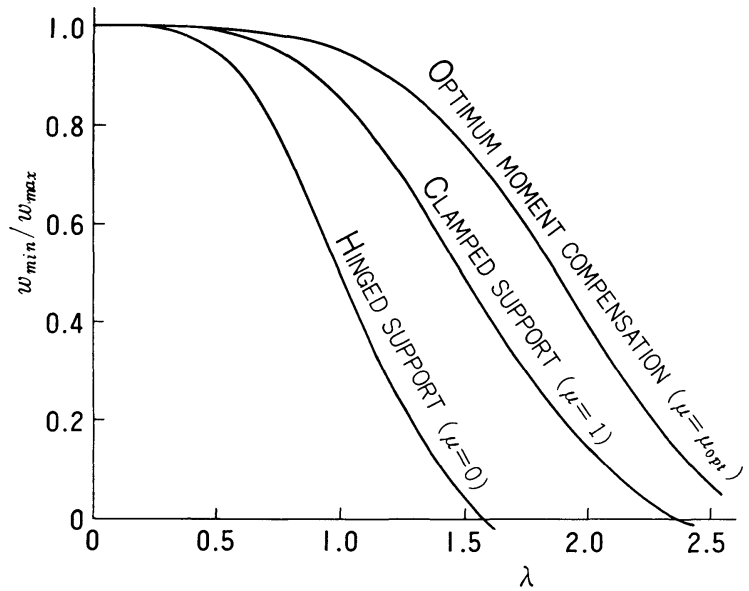

Fig. 6 Uniformity Index of Load Distribution of Covered Rollers of Optimum Moment Compensation, Clamped Support and Hinged Support

of Fig. 5, and compared with those of the hinged and clamped-support covered rollers without compensating moment. The uniformity index of load distribution is kept greater than 0.9 up to a value of $\lambda$ as high as 1 .

The effect of compensating moment on load distribution is shown in Fig. 7 for covered rollers of $\lambda=0.6$. When $\mu=0$, the load distribution is that of the hinged-support rollers, and is convex downwards. With an increase in compensating moment, the uniformity of load distribution is ameliorated better and better. An inflection point appears on the load distribution curve. When $\mu \fallingdotseq 9 / 7$, the effect of compensation is the maximum. The advantage of the moment compensation method over the clamped support comes from 


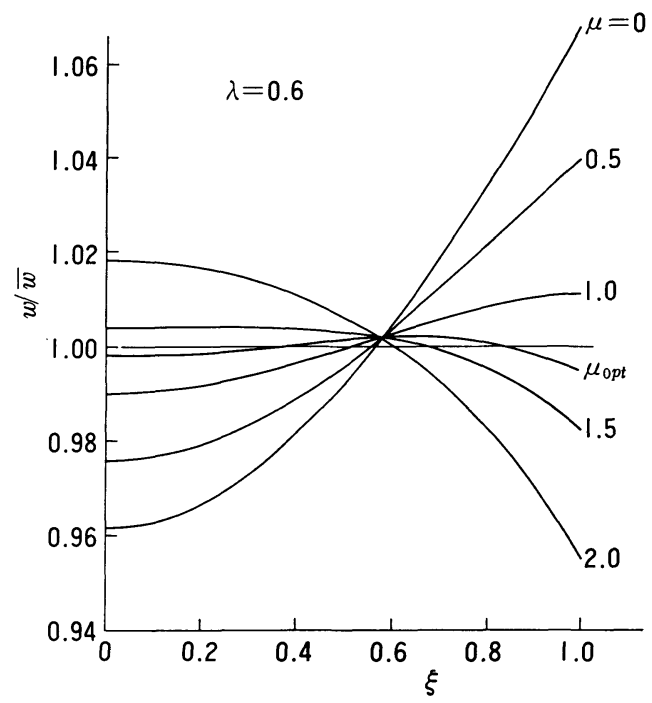

Fig. 7 Effect of Compensating Moment on Load Distribution

the difference of $\mu_{o p t} \fallingdotseq 9 / 7$ and $\mu=1$ for clamped-support rollers. For the values of $\mu$ beyond $\mu_{o p t}$, the load distribution curve is convex upwards, injuring the nip uniformity.

\section{Theoretical Analysis of Bending of Intermediate-} Support Pressure Rollers with Uniform Covering

Another means to make the load distribution uniform is to support the covered rollers at some intermediate positions between the ends and the center, instead of at both ends. It is evident from the theory of beam flexure that the maximum deflection of the beams of intermediate supoort is smaller than that of the beams supported at both ends. This suggests that the load distribution is more uniform when the covered rollers are supported at some intermediate positions.

The basic equations for the flexure of the intermediatesupport covered rollers are given by eqs. (3.2), (3.3) and the following equations:

$$
\left.\begin{array}{lr}
E_{1} I_{1}\left(d^{4} y / d x^{4}\right)=w, & |x| \leqq L_{2} \\
E_{2} I_{2}\left(d^{4} y / d x^{4}\right)=w, & L_{2} \leqq|x| \leqq L
\end{array}\right\}
$$

making use of the sign conventions shown in Fig. 8. Substituting eqs. (3.2) and (3.3) into eq. (7.1), and putting

$$
\left.\begin{array}{l}
\lambda_{1}{ }^{4}=\left(k / u_{c}\right) /\left(4 E_{1} I_{1} / L^{4}\right) \\
\lambda_{2}{ }^{4}=\left(k / u_{c}\right) /\left(4 E_{2} I_{2} / L^{4}\right)
\end{array}\right\}
$$

eq. (7.1) is converted into dimensionless form:

$$
\left.\begin{array}{lr}
d^{4} \zeta / d \xi^{4}+4 \lambda_{1}{ }^{4} \zeta=4 \lambda_{1}{ }^{4}\left(u_{c} / \bar{y}\right), & |\xi| \leqq \sigma \\
d^{4} \zeta / d \xi^{4}+4 \lambda_{2}{ }^{4} \zeta=4 \lambda_{2}{ }^{4}\left(u_{c} / \bar{y}\right), & \sigma \leqq|\xi| \leqq 1
\end{array}\right\}
$$

where

$$
\sigma=L_{2} / L
$$

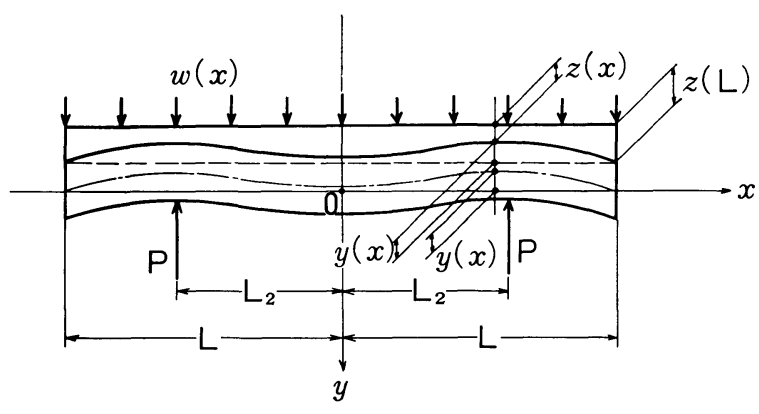

Fig. 8 Equivalent Mechanical Model of IntermediateSupport Covered Rollers

The general solutions of eq. (7.3) are:

$$
\left.\begin{array}{r}
\zeta=u_{c} / \vec{y}+A \dot{\psi}_{1}\left(\lambda_{1} \xi\right)+B \dot{\psi}_{2}\left(\lambda_{1} \xi\right)+C \psi_{1}\left(\lambda_{1} \xi\right)+D \psi_{2}\left(\lambda_{1} \xi\right), \\
|\xi| \leqq \sigma \\
\zeta=u_{c} / \bar{y}+F \dot{\psi}_{1}\left(\lambda_{2} \xi\right)+G \dot{\psi}_{2}\left(\lambda_{2} \xi\right)+H \psi_{1}\left(\lambda_{2} \xi\right)+J \psi_{2}\left(\lambda_{2} \xi\right), \\
\sigma \leqq \xi \leqq 1 \\
\zeta=u_{c} / \bar{y}+F^{\prime} \dot{\psi}_{1}\left(\lambda_{2} \xi\right)+G^{\prime} \dot{\psi}_{2}\left(\lambda_{2} \xi\right)+H^{\prime} \psi_{1}\left(\lambda_{2} \xi\right)+J^{\prime} \psi_{2}\left(\lambda_{2} \xi\right), \\
-1 \leqq \xi \leqq-\sigma
\end{array}\right\}
$$

From the condition of symmetry, $\zeta(\xi)$ should be an even function of $\xi$. Thus

$$
C=D=0, F=F^{\prime}, G=G^{\prime}, H=-H^{\prime}, J=-J^{\prime}
$$

From the practical consideration that the clamped support is almost impossible to manufacture for intermediatesupport covered rollers, only hinged-support rollers are dealt with. Hence the boundary conditions associated with the basic equations are expressed by

$$
\begin{aligned}
& y\left(L_{2-}\right)=y\left(L_{2+}\right), \quad E_{1} I_{1} y^{\prime \prime}\left(L_{2-}\right)=E_{2} I_{2} y^{\prime \prime}\left(L_{2+}\right), \\
& y^{\prime}\left(L_{2-}\right)=y^{\prime}\left(L_{2+}\right), \quad E_{1} I_{1} y^{\prime \prime \prime}\left(L_{2-}\right)=E_{2} I_{2} y^{\prime \prime \prime}\left(L_{2+}\right)+P \\
& y^{\prime \prime}(L)=0, \quad y^{\prime \prime \prime}(L)=0 \quad \text { at } \quad x=L
\end{aligned}
$$

wnere $L_{2+}$ means that $x \rightarrow L_{2}$ keeping $x \geqq L_{2}$, and $L_{2-}$ means that $x \rightarrow L_{2}$ keeping $x \leqq L_{2}$. These six conditions determine the six integration constants $A, B, F, G, H, J$ in eq. (7.5) together with eq. (7.6). They are the solution of the following simultaneous equation:

$$
\left(\begin{array}{ccl}
0 & 0 & -\dot{\phi}_{2}\left(\lambda_{2}\right) \\
0 & 0 & 2 \lambda_{2}{ }^{2} \psi_{1}\left(\lambda_{2}\right) \\
-\dot{\psi}_{1}\left(\lambda_{1} \sigma\right) & -\dot{\psi}_{2}\left(\lambda_{1} \sigma\right) & \dot{\phi}_{1}\left(\lambda_{2} \sigma\right) \\
2 \lambda_{1}{ }^{2} \psi_{2}\left(\lambda_{1} \sigma\right) & -2 \lambda_{1}{ }^{2} \psi_{1}\left(\lambda_{1} \sigma\right) & -2 \lambda_{2}{ }^{2} \psi_{2}\left(\lambda_{2} \sigma\right) \\
\lambda_{1}{ }^{2} \dot{\psi}_{2}\left(\lambda_{1} \sigma\right) & -\lambda_{1}{ }^{2} \dot{\psi}_{1}\left(\lambda_{1} \sigma\right) & -\beta \lambda_{2}{ }^{2} \dot{\psi}_{2}\left(\lambda_{2} \sigma\right) \\
-2 \lambda_{1}{ }^{4} \psi_{1}\left(\lambda_{1} \sigma\right) & -2 \lambda_{1}{ }^{4} \psi_{2}\left(\lambda_{1} \sigma\right) & 2 \beta \lambda_{2}{ }^{4} \psi_{1}\left(\lambda_{2} \sigma\right)
\end{array}\right.
$$




$$
\begin{array}{lll}
\dot{\phi}_{1}\left(\lambda_{2}\right) & -\phi_{2}\left(\lambda_{2}\right) & \phi_{1}\left(\lambda_{2}\right) \\
2 \lambda_{2}{ }^{2} \psi_{2}\left(\lambda_{2}\right) & \dot{\psi}_{2}\left(\lambda_{2}\right) & -\dot{\psi}_{1}\left(\lambda_{2}\right) \\
\dot{\psi}_{2}\left(\lambda_{2} \sigma\right) & \phi_{1}\left(\lambda_{2} \sigma\right) & \phi_{2}\left(\lambda_{2} \sigma\right) \\
2 \lambda_{2}{ }^{2} \psi_{1}\left(\lambda_{2} \sigma\right) & \dot{\psi}_{1}\left(\lambda_{2} \sigma\right) & \dot{\psi}_{2}\left(\lambda_{2} \sigma\right) \\
\beta \lambda_{2}{ }^{2} \dot{\psi}_{1}\left(\lambda_{2} \sigma\right) & -\beta \lambda_{2}{ }^{2} \psi_{2}\left(\lambda_{2} \sigma\right) & \beta \lambda_{2}{ }^{2} \psi_{1}\left(\lambda_{2} \sigma\right) \\
2 \beta \lambda_{2}{ }^{4} \psi_{2}\left(\lambda_{2} \sigma\right) & \beta \lambda_{2}{ }^{2} \dot{\psi}_{2}\left(\lambda_{2} \sigma\right) & -\beta \lambda_{2}{ }^{2} \dot{\psi}_{1}\left(\lambda_{2} \sigma\right)
\end{array}
$$$$
\left(\begin{array}{l}
A \\
B \\
F \\
G \\
H \\
J
\end{array}\right)=2 \lambda_{1} 4 \cdot\left(\begin{array}{l}
0 \\
0 \\
0 \\
0 \\
0 \\
1
\end{array}\right)
$$

Introducing the solution of eq. (7.8) into eq. (7.5), the following equations for the deflection and the load distribution are obtained:

$$
\begin{aligned}
& y / \vec{y}=A \dot{\phi}_{1}\left(\lambda_{1} \xi\right)+B \dot{\phi}_{2}\left(\lambda_{1} \xi\right) \\
& -F \dot{\phi}_{1}\left(\lambda_{2}\right)-G \dot{\phi}_{2}\left(\lambda_{2}\right)-H \psi_{1}\left(\lambda_{2}\right)-J \psi_{2}\left(\lambda_{2}\right), 0 \leqq \xi \leqq \sigma \\
& y / \bar{y}=F \dot{\phi}_{1}\left(\lambda_{2} \xi\right)+G \dot{\psi}_{2}\left(\lambda_{2} \xi\right)+H \psi_{1}\left(\lambda_{2} \xi\right)+J \psi_{2}\left(\lambda_{2} \xi\right) \\
& \left.-F \dot{\phi}_{1}\left(\lambda_{2}\right)-G \dot{\phi}_{2}\left(\lambda_{2}\right)-H \psi_{1}\left(\lambda_{2}\right)-J \psi_{2}\left(\lambda_{2}\right), \sigma \leqq \xi \leqq 1\right) \\
& w / \bar{w}=-A \dot{\psi}_{1}\left(\lambda_{1} \xi\right)-B \dot{\psi}_{2}\left(\lambda_{1} \xi\right), \quad \quad \cdots \cdots \cdots \cdots \cdots \\
& w / \bar{w}=-F \dot{\psi}_{1}\left(\lambda_{2} \xi\right)-G \dot{\psi}_{2}\left(\lambda_{2} \xi\right)-H \psi_{1}\left(\lambda_{2} \xi\right)-J \psi_{2}\left(\lambda_{2} \xi\right), \\
& \sigma \leqq \xi \leqq 1
\end{aligned}
$$

\section{Numerical Results and Discussions}

Among the factors which determine the load distribution, the position of supports $L_{2}=\sigma L$ and the ratio of flexural rigidity $\beta=E_{2} I_{2} / E_{1} I_{1}$ can be arbitrarily chosen as the most important ones for the flexural behavior of the intermediate-support covered rollers. Here the effect of $\sigma$ is investigated exclusively, keeping $\beta$ constant. That of the rigidity ratio will be discussed elsewhere. Load distributions are calculated for hinged-support rollers of uniform mandrel and with uniform covering. Numerical calculations are carried out making use of electronic digital computer.

Fig. 9 depicts how the load distributions are altered by changing the intermediate support position for a fixed value of $\lambda=0.6$. The condition of $\sigma=1$ signifies that the covered rollers are supported at both ends, and $\sigma=0$ means that they are supported at the center. Though the load distributions for these extreme cases are omitted in Fig. 9, their uniformity of load distribution is inferior to those of the intermediate-support covered rollers. As the support position becomes more distant from that of these

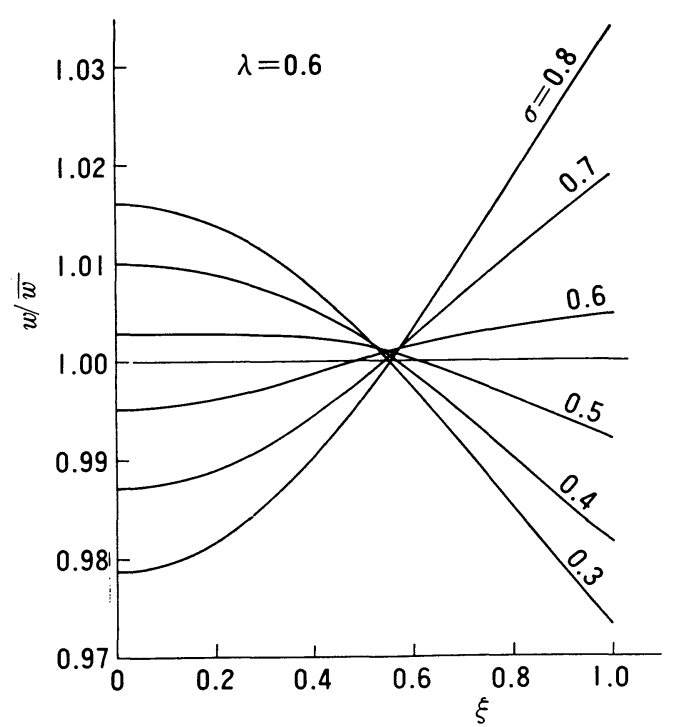

Fig. 9 Effect of Support Position on Load Distribution

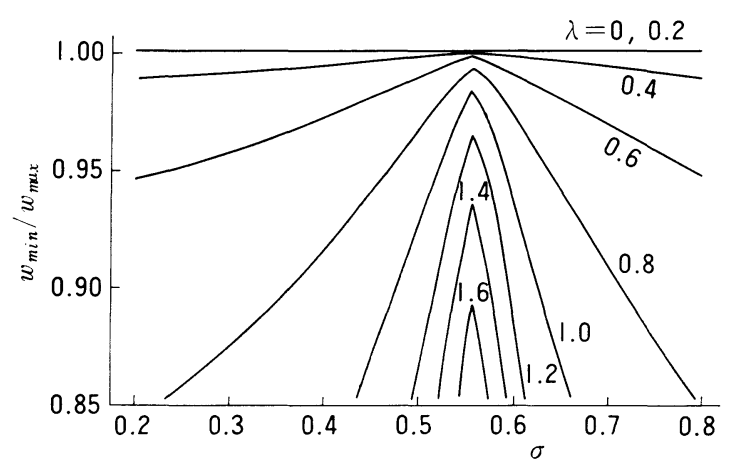

Fig. 10 Effect of Support Position on Uniformity Index of Load Distribution of Intermediate-Support Covered Rollers

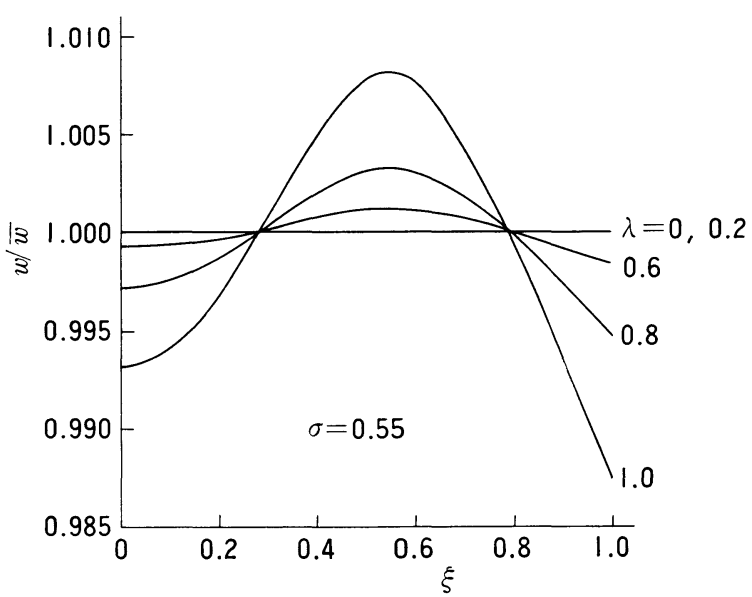

Fig. 11 Load Distribution, Covered Rollers Supported at Optimum Positions 
extreme cases, more uniform load distribution is provided. The most favorable uniformity index is reached for a value of $\sigma$ between 0.5 and 0.6 .

To grasp the behavior more clearly, the uniformity indices of load distribution are plotted against $\sigma$ for several values of $\lambda$ in Fig. 10. Although the optimum values of $\sigma$ vary slightly for different values of $\lambda$, they are nearly equal to 0.55 . Load distribution curves for $\sigma=0.55$ are plotted for various values of $\lambda$ in Fig. 11. The load per unit length of the covered roller takes the minimum at $\xi=0$, the maximum at $\xi \doteqdot \sigma$, and the smallest values at $\xi=1$, irrespective of the value of $\lambda$.

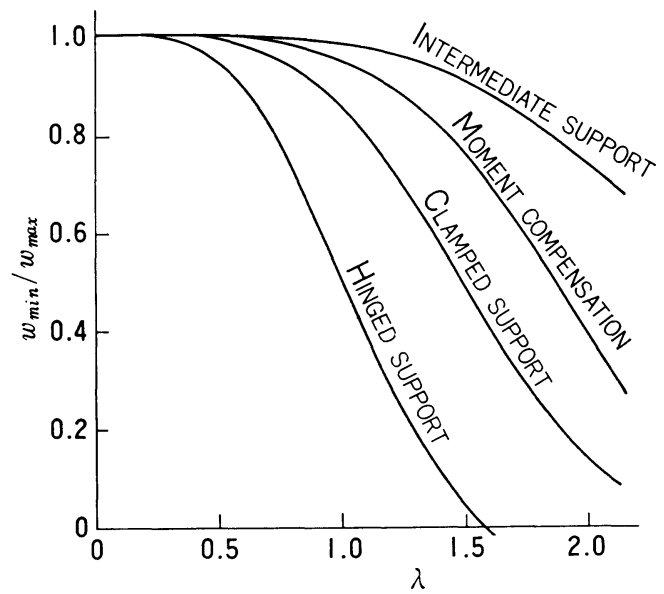

Fig. 12 Uniformity Index of Load Distribution of Covered Rollers of Intermediate Support, Moment Compensation, Clamped Support and Hinged Support

Fig. 12 compares schematically the uniformity indices of load distribution of the intermediate-support rollers of $\sigma=0.55$ with those of the covered rollers supported at both ends and with those of the moment-compensated covered rollers.

A glance at Fig. 12 explains the superiority of the intermediate support method in providing uniform load distribution. It keeps the uniformity index of 0.9 up to a value of $\lambda$ as high as about 1.5. If $\sigma$ is not equal to the optimum value, the nip uniformity is certainly deteliorated.

\section{Conclusions}

The theoretical investigations explained above are summarized into the following conclusions:

(1) The basic equations of flexure of a pair of mechani- cally similar rubber-covered pressure rollers, derived in the earlier paper, determine the camber distribution of the covering, provided the load distribution is given. The camber distribution of the covering which provides a uniformly distributed load is calculated. The cambering thus obtained is proved to be the same as the deflection of the roller mandrel under the given uniformly distributed load, and its magnitude is a function of the design load and of other several factors.

(2) Once the optimum thickness distribution of the covering is determined for one loading condition of a mangle, the load distribution becomes uneven when the mangle works under other loading conditions. This type of uneveness of load distribution is very sensitive to the deviation of the actual load from the design load. However, the uniformity index of load distribution when the covered rollers with the optimum thickness distribution are used under the mean load higher than the design load is always closer to 1 than that when the pressure rollers with covering of uniform thickness are used under the same mean load.

(3) Theoretical analysis of moment compensation method has revealed that there exists an optimum amount of compensating moment which is proportional to a product of $P$ times $L$ and is almost independent of $\lambda$ in a range of $\lambda$ below 1.0. If this optimum compensating moment is applied to the covered rollers at both ends, the uniformity index of load distribution is greatly ameliorated. The covered rollers with moment compensation mechanism excel the clamped-support covered rollers in nip uniformity.

(4) Intermediate-support covered rollers are the best in nip uniformity when they are supported at an optimum position. This optimum position of intermediate support is about 55 percent of the half of the length of the covered rollers from the center, and is independent of the characteristic number $\lambda$ and of the average load $\bar{w}$. If the position of the intermediate support differs from the optimum, the nip uniformity is deteriorated, particularly when the characteristic number $\lambda$ is large.

The numerical calculations in this study were carried out by the electronic digital computer of the computer center of the Tokyo University.

\section{References}

[1] K. Nakajima, S. Tsujio: J. Text. Mach. Soc. Japan, 16, 26 (1970)

[2] G. J. Parish: Brit. J. Appl. Phys., 12, 333 (1961)

[3] M. Jukich: Modern Plastics, 33, No. 8, 138 (1956)

[4] E. Moss: J. Text. Inst., 50, P654 (1959) 